\title{
BMJ Open Extrapulmonary effects of continuous airway pressure on patients with obstructive sleep apnoea: protocol for an overview of systematic reviews
}

\author{
Gonzalo Labarca, ${ }^{1,2,3,4}$ Francisco Ortega, ${ }^{5}$ Alex Arenas, ${ }^{5}$ Tomas Reyes, ${ }^{5}$ \\ Gabriel Rada, ${ }^{3,4}$ Jorge Jorquera ${ }^{6}$
}

To cite: Labarca G, Ortega F, Arenas A, et al. Extrapulmonary effects of continuous airway pressure on patients with obstructive sleep apnoea: protocol for an overview of systematic reviews. BMJ Open 2017;7:e015315. doi:10.1136/ bmjopen-2016-015315

- Prepublication history and additional material for this paper are available online. To view these files please visit the journal online (http://dx.doi. org/10.1136/bmjopen-2016015315).

Received 25 November 2016 Revised 24 May 2017 Accepted 5 June 2017

\section{CrossMark}

${ }^{1}$ Facultad de Medicina, Universidad San Sebastian, Concepcion, Chile

${ }^{2}$ Complejo Asistencial Dr. Victor Rios Ruiz, Los Angeles, Chile

${ }^{3}$ Faculty of Medicine, Evidence Based Health Care Program, Pontificia Universidad Católica de Chile, Santiago, Chile

${ }^{4}$ Epistemonikos Foundation,

Santiago, Chile

${ }^{5}$ Department of Internal Medicine, Faculty of Medicine, Pontificia Universidad Católica de Chile, Santiago, Chile

${ }^{6}$ Sleep Center and Respiratory Diseases, Clinica Las Condes, Santiago, Chile

Correspondence to

Dr Gonzalo Labarca; glabarcat@ gmail.com

\section{ABSTRACT}

Introduction Obstructive sleep apnoea (OSA) is associated with both pulmonary and extrapulmonary consequences. Continuous positive airway pressure (CPAP) is an effective treatment for pulmonary symptoms. However, it is not clear if CPAP modifies extrapulmonary symptoms, especially in the long term. To date, several studies addressing this issue have been performed, but the evidence is scattered across different reviews that address each outcome separately. Therefore, we will conduct an overview to assess and summarise all systematic reviews on the extrapulmonary effects of this intervention in patients with OSA.

Methods This is a protocol for a systematic overview of reviews that will follow the guidelines from the Cochrane Handbook of Systematic Reviews of Interventions and PRISMA-P. We will conduct comprehensive literature searches using MEDLINE, EMBASE, Cochrane, CINAHL, DARE and Epistemonikos. We will consider systematic reviews and meta-analyses of randomised controlled trials evaluating the extrapulmonary effects of CPAP on OSA. Two reviewers will independently screen articles for inclusion using criteria that are determined a priori, assessing the quality of included reviews and extracting relevant information. We will create matrices of evidence in the Epistemonikos database, including all systematic reviews addressing a similar question and all studies addressing the question in those reviews. We will try to explain why studies are not included in individual reviews using the inclusion criteria, and we will estimate the overlap between different reviews in the same matrix of evidence.

Ethics and dissemination This review will be published in a peer-reviewed journal.

Trial registration number CRD42014013036

\section{INTRODUCTION}

Obstructive sleep apnoea (OSA) is a chronic disease characterised by chronic intermittent hypoxia (CIH) secondary to an obstruction from the upper airways. ${ }^{1}$ This disease is highly prevalent. Epidemiological studies report a prevalence of $2 \%-9 \%$ for women and $4 \%-24 \%$ for men; this prevalence is

\section{Strengths and limitations of this study}

- This overview of systematic reviews will summarise a substantial body of evidence on the extrapulmonary effects of continuous positive airway pressure in patients with obstructive sleep apnoea, and it will provide a high-quality summary that is useful for clinicians and policy-makers.

- This overview of systematic reviews will include critical and non-critical outcomes to establish new connections between obstructive sleep apnoea and chronic conditions, such as diabetes and blood pressure.

- The main limitations are related to the quality, currency and coverage of the identified systematic reviews.

- Another limitation is that new randomised controlled trials might not be included in published systematic reviews. In those cases, an evidence matrix provided by Epistemonikos will include new evidence and interested readers will be notified.

higher in obese patients (more than $30 \mathrm{~kg} /$ $\mathrm{m}^{2}$ of body mass index) with a prevalence of $40 \% .^{23}$ The high-risk group includes patients with chronic arterial hypertension and hyperglycaemia. ${ }^{4}$ OSA severity varies from mild OSA, defined by an apnoea/hypopnea index (AHI) with 5-15 events/hour, to moderate (AHI 15-30 events/hour) and severe (>30 events/hour). ${ }^{56}$

Chronic hypoxia leads to several extrapulmonary changes, such as endothelial dysfunction, cardiovascular alterations, poor neurocognitive function and increases in the concentration of proinflammatory biomarkers. ${ }^{178}$ These changes increase the risk of stroke, acute myocardial infarction, heart failure, diabetes mellitus, dyslipidaemia and non-alcoholic fatty liver disease (NAFLD), and these related conditions may suggest a global metabolic effect with dysfunction of several organs. ${ }^{4}$ 
Another relevant issue regarding the OSA pathology is sleep fragmentation and changes in sleep structure. Sleep deprivation is associated with changes in the white matter, which can affect attention and vigilance. However, CIH may decrease the structure of the blood-brain barrier. ${ }^{9}$

OSA treatment includes several medical and non-medical interventions. ${ }^{10}$ For all patients with OSA, changes in sleepiness, physical activity and low weight therapies are suggested. Medical treatment includes the use of a continuous positive airway pressure (CPAP) device. CPAP increases the air flow, decreasing the number of upper airways that are collapsed and improving the effective sleep time. Current indications for CPAP include all severe OSA (AHI $>30$ events/ hour) and moderate OSA (AHI 15-30 events/hours) patients with high cardiovascular risk. ${ }^{16}$ On the one hand, this intervention has been shown to improve outcomes and decrease the cardiovascular risk and mortality in some observational studies. ${ }^{1011}$ On the other hand, this therapy is costly, and its widespread use would carry major implications for health systems. ${ }^{1}{ }^{1012}$ A substantial improvement in extrapulmonary consequences would be a strong argument for considering the use of CPAP in a broader range of patients and for intensifying the efforts to improve the early diagnosis of OSA. ${ }^{711}$

Current clinical guidelines are focused on patient-focused outcomes ${ }^{1613}$ despite the surrogate outcomes and biomarkers related to CHI. In addition, because of the available data, other clinical conditions, such as dyslipidaemia, NAFLD and insulin resistance, are not current indications for treating patients with moderate OSA. ${ }^{1}$

However, an overview of the review (or an umbrella review) is a novel methodological approach with a clear objective that is designed to answer a clinical question about a healthcare intervention via explicit, reproducible methods. The data are collected and presented in a user-friendly format. This methodological approach is different from clinical guidelines. ${ }^{14}$

We anticipate that the information on the effects of this intervention will be substantial, although fragmented into different studies and systematic reviews, based on the worldwide interest in this topic and the broad nature of health outcomes that can be affected. Therefore, an overview of systematic reviews can provide a comprehensive picture of both the evidence needed to make decisions regarding this topic and the research gaps in this area. ${ }^{15}$

\section{OBJECTIVES}

The objective of this overview of systematic reviews will be to evaluate the current evidence of the effects of CPAP treatment on extrapulmonary outcomes associated with OSA.

\section{METHODS}

This overview of systematic reviews will be performed according to recommendations of the Cochrane Collaboration $^{16}$ and an adaptation of the Preferred reporting items for systematic review and meta-analysis protocols PRISMA-P Statement, when pertinent. ${ }^{17}$

This overview of systematic reviews is registered in the PROSPERO registry: CRD42014013036.

\section{Eligibility and inclusion criteria}

We will consider systematic reviews and primary studies included in those reviews that satisfy the following criteria:

\section{Type of reviews}

In this overview, we will consider systematic reviews that summarise randomised controlled studies according to the following definition:

In accordance with the Cochrane Collaboration and the PRISMA Statement, 'a systematic review attempts to collate all empirical evidence that fits pre-specified eligibility criteria to answer a specific research question. It uses explicit, systematic methods that are selected with a view to minimising bias, thus providing reliable findings from which conclusions can be drawn and decisions made'. ${ }^{18}$

An eligible review will need to fulfil the following operational criteria:

- To report a search in at least one electronic database.

- To report at least one criterion for the inclusion of studies.

- To report a pooled measure of effect for at least one outcome.

- To evaluate the quality (risk of bias) of the included primary studies.

\section{Type of participants}

We will include reviews that summarise studies on adult patients with OSA.

\section{Type of intervention}

We will include reviews that evaluate the effects of CPAP compared with no treatment, medical therapy or sham CPAP.

\section{Types of outcomes}

We will include reviews evaluating studies reporting any of the following outcomes:

Primary outcomes: patient-important outcomes that are not related to the direct effects of CPAP on respiratory parameters or pulmonary function, such as

- Mortality.

- Cardiovascular diseases (different from pulmonary hypertension or right-side heart failure), including the development or worsening of systolic heart failure, acute myocardial infarction and stroke.

Secondary outcomes: surrogate outcomes that are considered good predictors of patient important outcomes.

- Development of hypertension or change in the blood pressure level.

- Development of diabetes mellitus type 2, glycaemic control or HbA1C levels.

- Neurological consequences (somnolence, sleepiness, cognitive function, occupational or driving accidents). 
We will also consider some surrogate outcomes whose roles are controversial, although they are extensively studied, such as homeostatic model assessment test, high-sensitivity C-reactive protein, tumor necrosis factor- $\alpha$ (TNF- $\alpha$ ) or interleukin-6 (IL-6) levels, leptin levels and lipid profile.

We will describe a subgroup analysis derived from systematic reviews according to the sleep apnoea severity, defined according to the AHI as mild, moderate or severe.

\section{Literature search and search strategy}

\section{Electronic searches}

We will conduct sensitive searches of MEDLINE, EMBASE, Cochrane Database of Systematic Reviews, CINAHL, Database of Abstract of Reviews of Effects (DARE) and Epistemonikos with no language or publication restrictions. A summary of the literature search is presented in online supplementary material 1 .

\section{Other sources}

The electronic search will be supplemented with the following:

- Review for additional relevant studies in the references of the included reviews.

- Review of the references of relevant narrative reviews and guidelines.

\section{Data collection and analysis \\ Selection of studies}

Two reviewers will independently screen titles and abstracts to identify relevant articles. The full text of potentially eligible reviews will be retrieved and independently evaluated by two authors for final inclusion. Disagreements will be addressed through discussion; if a consensus cannot be reached, a third author will resolve the disagreements.

\section{Data extraction}

Two reviewers will independently extract data from each included review using standardised forms. To ensure consistency, we will conduct calibration exercises before the review. Discrepancies in the extracted data will be resolved by discussion and, if needed, a third author will arbitrate.

We will extract the following information from the included systematic reviews:

Reference details: inclusion/exclusion criteria, design of included primary studies (randomised vs others), date of last search (if it is not described, we will count the date of the most recent study), risk of bias of assessment methods, meta-analysis and summary of findings table (GRADE).

We will also extract the list of included studies in the review.

\section{Quality assessment of systematic reviews}

We will evaluate the quality (risk of bias) of included systematic reviews through the AMSTAR measurement tool. ${ }^{19}$ Two reviewers will independently use the checklist, and discrepancies will be resolved by consensus.

\section{Data synthesis}

\section{Comparison between reviews}

To compare different reviews addressing similar questions, we will create a matrix of evidence in the Epistemonikos database. ${ }^{20} \mathrm{~A}$ matrix of evidence is a tabular approach for displaying the cluster of systematic reviews that share included studies. It includes all systematic reviews addressing a similar question (ie, sharing at least one included study) and all studies addressing the question in those reviews. ${ }^{21}$ The table will be created independently by two reviewers, and differences will be resolved by consensus.

Finally, a summary of results and evidence grading will be performed using the GRADE method. We evaluate the evidence derived from randomised controlled trial (RCT) or observational studies, and we will downgrade or upgrade this evidence according to the GRADE approach. ${ }^{22}$ A summary of findings table will be created using GRADEpro software.

\section{Comparison of primary studies included in reviews}

We will try to explain why studies are not included in individual reviews using the following criteria:

- The study was published after the date of the last search of the review.

- The study is mentioned as an excluded study in the review.

- The study is not mentioned as an excluded study, but it does not meet the inclusion criteria of the review.

- The study was missed in the review.

- Other (eg, studies awaiting assessment).

- Overlap between reviews.

We will estimate the overlap between different reviews in the same matrix of evidence using the covered area and corrected covered area (CCA) methods. A CCA $\geq 15$ will be considered a very high level of overlap. ${ }^{23}$

\section{DISCUSSION}

This overview of systematic reviews will summarise a substantial body of evidence addressing the effects of CPAP on a wide array of non-pulmonary outcomes.

We decided to include evidence from systematic reviews that contain RCTs for critical primary and secondary outcomes (eg, heart failure, blood pressure, long-term mortality and cardiovascular mortality) and systematic reviews derived for some specific outcomes (ie, surrogate outcomes, such as TNF- $\alpha$ and IL-6). In addition, the GRADE approach would downgrade or upgrade the evidence derived from RCT studies included in systematic reviews.

One of the limitations of this overview is the gap between systematic reviews and RCTs that have recently been published. Some of the biggest CPAP trials have just recently been reported, but they have not yet been 
evaluated in a systematic review or meta-analysis. In those cases, the evidence matrix provided by the Epistemonikos database will include new evidence and interested readers will be automatically notified of updates. ${ }^{20} 21$

Finally, this information will provide decision-makers with data at both the individual and policy levels and will help identify research gaps.

\section{ETHICS AND DISSEMINATION}

Ethics approval is not required. This review will be published in a peer-reviewed journal after a peer-review process. The results will provide information for future studies that clinicians could use to make individual decisions and that healthcare managers, administrators and policymakers could use to guide resource allocation.

Contributors GR: conception and design of the study, acquisition of data, data analysis, preparation of the manuscript and final proofread. Technical support and contact with Epistemonikos database. GL: conception and design of the study, acquisition of data, data analysis, preparation of the manuscript and final proofread. Register in PROSPERO record. FO: acquisition of data and data analysis, preparation of the manuscript, critical analysis and final proofread. AA: acquisition of data and data analysis, preparation of the manuscript, critical analysis and final proofread. TR: acquisition of data and data analysis, preparation of the manuscript, critical analysis and final proofread. JJ: data analysis, critical analysis, manuscript correction and final proofread.

Competing interests None declared.

Provenance and peer review Not commissioned; externally peer reviewed. Data sharing statement None.

Open Access This is an Open Access article distributed in accordance with the Creative Commons Attribution Non Commercial (CC BY-NC 4.0) license, which permits others to distribute, remix, adapt, build upon this work non-commercially, and license their derivative works on different terms, provided the original work is properly cited and the use is non-commercial. See: http://creativecommons.org/ licenses/by-nc/4.0/

(c) Article author(s) (or their employer(s) unless otherwise stated in the text of the article) 2017. All rights reserved. No commercial use is permitted unless otherwise expressly granted.

\section{REFERENCES}

1. Qaseem A, Holty JE, Owens DK, et al. Clinical Guidelines Committee of the American College of Physicians. Management of obstructive sleep apnea in adults: a clinical practice guideline from the American College of Physicians. Ann Intern Med 2013;159:471-83.

2. Heinzer R, Vat S, Marques-Vidal P, et al. Prevalence of sleepdisordered breathing in the general population: the HypnoLaus study. Lancet Respir Med 2015;3:310-8.
3. Young T, Palta M, Dempsey J, et al. The occurrence of sleepdisordered breathing among middle-aged adults. $N$ Engl J Med 1993;328:1230-5.

4. Sánchez-de-la-Torre M, Campos-Rodriguez F, Barbé F. Obstructive sleep apnoea and cardiovascular disease. Lancet Respir Med 2013;1:61-72.

5. Qaseem A, Dallas P, Owens DK, et al. Diagnosis of obstructive sleep apnea in adults: a clinical practice guideline from the American College of Physicians. Ann Intern Med 2014;161:210-20.

6. Lloberes P, Durán-Cantolla J, Martínez-García MÁ, et al. Diagnosis and treatment of sleep apnea-hypopnea syndrome. Spanish Society of Pulmonology and Thoracic Surgery. Arch Bronconeumol 2011:47:143-56.

7. Labarca G, Cruz N R, Descalzi F. [Multisystemic involvement in obstructive sleep apnea]. Rev Med Chil 2014;142:748-57.

8. Vicente E, Marin JM, Carrizo SJ, et al. Upper airway and systemic inflammation in obstructive sleep apnoea. Eur Respir $J$ 2016;48:1108-17.

9. Gildeh N, Drakatos P, Higgins S, et al. Emerging co-morbidities of obstructive sleep apnea: cognition, kidney disease, and cancer. $J$ Thorac Dis 2016;8:E901-E917.

10. Calik MW. Treatments for obstructive sleep apnea. J Clin Outcomes Manag 2016;23:181-92

11. Marin JM, Carrizo SJ, Vicente E, et al. Long-term cardiovascular outcomes in men with obstructive sleep apnoea-hypopnoea with or without treatment with continuous positive airway pressure: an observational study. Lancet 2005;365:1046-53.

12. Català R, Villoro R, Merino $\mathrm{M}$, et al. Cost-effectiveness of continuous positive airway pressure treatment in moderate-severe obstructive sleep apnea syndrome. Arch Bronconeumol 2016;52:461-9.

13. Strohl KP, Brown DB, Collop N, et al. An official American Thoracic Society Clinical Practice Guideline: sleep apnea, sleepiness, and driving risk in noncommercial drivers. An update of a 1994 Statement. Am J Respir Crit Care Med 2013;187:1259-66.

14. Pollock M, Fernandes RM, Becker LA, et al. What guidance is available for researchers conducting overviews of reviews of healthcare interventions? A scoping review and qualitative metasummary. Syst Rev 2016;5:190.

15. Thomson D, Russell K, Becker L, et al. The evolution of a new publication type: steps and challenges of producing overviews of reviews. Res Synth Methods 2010;1(3-4):198-211.

16. Collaboration" TC. In: Higgins J, Green S, eds. Cochrane Handbook for Systematic Reviews of Interventions Version 5.1.0 [updated March 2011], 2011.

17. Moher D, Shamseer L, Clarke M, et al. Preferred reporting items for systematic review and meta-analysis protocols (PRISMA-P) 2015 statement. Syst Rev 2015;4:1.

18. Oxman $A D$, Guyatt $\mathrm{GH}$. The science of reviewing research. Ann $N Y$ Acad Sci 1993;703:125-34.

19. Shea BJ, Grimshaw JM, Wells GA, et al. Development of AMSTAR: a measurement tool to assess the methodological quality of systematic reviews. BMC Med Res Methodol 2007;7:10.

20. Rada G, Pérez D, Capurro D. Epistemonikos: a free, relational, collaborative, multilingual database of health evidence. Stud Health Technol Inform 2013;192:486-90.

21. Rada G, Neumann I, Cerda J. A new way to find evidence quickly and efficiently. Medwave 2014;14:e6044.

22. Guyatt GH, Oxman AD, Vist GE, et al. GRADE: an emerging consensus on rating quality of evidence and strength of recommendations. BMJ 2008;336:924-6.

23. Pieper D, Antoine SL, Mathes T, et al. Systematic review finds overlapping reviews were not mentioned in every other overview. $J$ Clin Epidemiol 2014;67:368-75. 